\title{
RF Modeling of p-n-p-n Double-Gate Tunneling Field-Effect Transistors
}

\author{
Saeid Marjani* and Seyed Ebrahim Hosseini \\ Department of Electrical Engineering \\ Ferdowsi University of Mashhad \\ Mashhad, Iran \\ *saeid.marjani@stu.um.ac.ir \\ ehosseini@um.ac.ir
}

\begin{abstract}
This paper presents radio-frequency (RF) modeling of p-n-p-n double-gate tunneling field-effect transistors (TFETs). The p-n-p-n TFETs are evaluated for various RF parameters such as cut-off frequency, maximum oscillation frequency, gatesource capacitance, gate-drain capacitance, channel resistance, and transconductance. Direct comparisons of high-frequency performances and extracted parameters are made with conventional TFETs. A nonquasistatic radio-frequency model has been used, along with SPICE simulations to investigate p-np-n TFETs with RF parameters extracted from TCAD simulation Y-parameters. The results show excellent agreements with the TCAD simulation results for the high frequency range up to the cut-off frequency for the millimeter-wave applications.
\end{abstract}

Keywords- Modeling; Nonquasistatic (NQS); Radio-frequency (RF); p-n-p-n; Tunneling field-effect transistor (TFET).

\section{INTRODUCTION}

Today, steep sub-threshold slope (SS), very low leakage current and suppressed short channel effects (SCE) are major requirement for energy efficient digital ICs. In response to the enormous challenges associated with scaled MOSFETs, bandto-band tunneling (BTBT) field-effect transistors (TFETs) have been proposed to replace MOSFET for ultralow power applications [1-3]. The basic structure for a TFET is gate reverse biased $\mathrm{p}-\mathrm{i}-\mathrm{n}$ diode with heavily doped source and drain regions. Although, TFETs have shown better characteristics than MOSFETs in terms of low sub-threshold swing and low OFF-current but their low ON-current is problematic. In recent years, numerous experimental and simulation based techniques have been proposed in order to improve tunneling probability and ON-current of silicon-based TFETs such as band-gap engineering [4, 5], small band-gap materials [6], high-k dielectric materials [7], pocket doping [8], extended source [9, $10]$ and $p-n-p-n$ structure $[11,12]$. Although there have been many experimental and simulation studies on the design, and drain current model of p-n-p-n TFETs $[11,12]$ but their RF modeling has been seldom reported. In this work, a radiofrequency (RF) model for $p-n-p-n$ double-gate TFETs is presented and their RF performances have been compared with that of conventional TFETs. The small-signal parameters were extracted from the analytical equations of the Y-parameters of a nonquasistatic (NQS) radio-frequency model for analysis of cut-off frequency, maximum oscillation frequency, gate-source

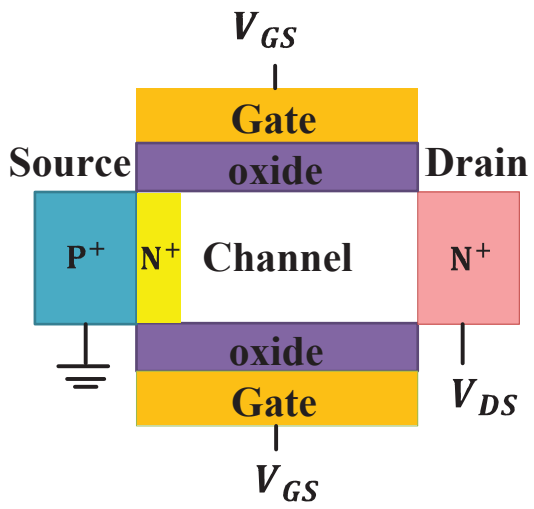

Fig. 1. Cross-section views of the p-n-p-n TFET. Parameters: channel length $\mathrm{L}_{\mathrm{ch}}=45 \mathrm{~nm}$, pocket width $\mathrm{L}_{\mathrm{p}}=5 \mathrm{~nm}$, oxide thickness $\left(\mathrm{t}_{\mathrm{ox}}\right)=2 \mathrm{~nm}, \mathrm{t}_{\mathrm{si}}=15 \mathrm{~nm}$, Source doping $\mathrm{N}_{\mathrm{A}}=1 \times 10^{20} \mathrm{~cm}^{-3}$, drain doping $\mathrm{N}_{\mathrm{D}}=1 \times 10^{18} \mathrm{~cm}^{-3}, \mathrm{~N}_{\mathrm{ch}}(\mathrm{p})=$ $1 \times 10^{15} \mathrm{~cm}^{-3}$, pocket doping $\left(\mathrm{N}_{\mathrm{p}}\right)=5 \times 10^{19} \mathrm{~cm}^{-3}$.

capacitance, gate-drain capacitance, channel resistance, and transconductance. The paper is organized as follows: In Sec. II, we have explained device structure and simulation. The radiofrequency model for $p-n-p-n$ TFETs is explained in Sec. III. In Sec. IV, we discuss the RF performances of $p-n-p-n$ and conventional TFETs, and obtained results are presented. In section $\mathrm{V}$, validation of the radio-frequency model is investigated. Finally, Sec. VI concludes the work.

\section{DeVICE Structure AND Simulation}

Fig. 1 shows cross-sectional views of the Si based doublegate $\mathrm{p}-\mathrm{n}-\mathrm{p}-\mathrm{n}$ TFETs structure used in the two-dimensional device simulations [13]. A double-gate geometry is considered with Si-channel doping concentration $N A=1 \times 10^{15} \mathrm{~cm}^{-3}$, channel length of $45 \mathrm{~nm}$, silicon film thickness of $15 \mathrm{~nm}$, high$\mathrm{k}$ gate oxide thickness of $2 \mathrm{~nm}\left(\varepsilon_{\mathrm{ox}}=29\right)$. The device is uniformly doped at the source and drain regions with concentrations $1 \times 10^{20} \mathrm{~cm}^{-3}$ and $1 \times 10^{18} \mathrm{~cm}^{-3}$, respectively. For $\mathrm{p}-\mathrm{n}-\mathrm{p}-\mathrm{n}$ structure, an $\mathrm{n}^{+}$heavily doped pocket of width $5 \mathrm{~nm}$ is used. The $\mathrm{n}^{+}$pocket doping concentrations is taken $5 \times 10^{19} \mathrm{~cm}^{-3}$. For higher accuracy, band-to-band tunneling has been modeled using a dynamic nonlocal path tunneling approach for the device performance calculations. Along with nonlocal BTBT model, the physical models activated during device simulations are: Shockley-Read-Hall recombination model, auger 
recombination, concentration and field dependent mobility models, hurkx recombination model and band gap narrowing [14].

\section{RADIO-FREQUENCY MODEL OF P-N-P-N TFETS}

The intrinsic nonquasistatic (NQS) equivalent circuit of a transistor to extract small-signal parameters of p-n-p-n TFETs is shown in Fig. 2. In the circuit, $C_{g s}$ and $C_{g d}$ are intrinsic gatesource and gate-drain capacitances. $R_{g}$ is the effective gate resistance. $C_{s d}$ is source-drain capacitance which varies with the drain bias of short channel devices. Time constant $\tau, g_{m}$ and $g_{d s}$ are the charge transport delay, transconductance and sourcedrain conductance, respectively. The $Y$-parameters of the intrinsic nonquasistatic equivalent circuit are expressed as follows [15]:

$$
\begin{aligned}
& \mathrm{Y}_{11}=\frac{\omega^{2} R_{g}\left(C_{g s}+C_{g d}\right)^{2}+j \omega\left(C_{g s}+C_{g d}\right)}{1+\omega^{2} R_{g}^{2}\left(C_{g s}+C_{g d}\right)^{2}} \\
& \mathrm{Y}_{12}=\frac{-\omega^{2} R_{g} C_{g d}\left(C_{g s}+C_{g d}\right)-j \omega C_{g d}}{1+\omega^{2} R_{g}^{2}\left(C_{g s}+C_{g d}\right)^{2}} \\
& \mathrm{Y}_{21}=\frac{-j \omega C_{g d}-\omega^{2} R_{g} C_{g d}\left(C_{g s}+C_{g d}\right)}{1+\omega^{2} R_{g}^{2}\left(C_{g s}+C_{g d}\right)^{2}} \\
& +\frac{g_{m}-j \omega g_{m}\left[\tau+R_{g}\left(C_{g s}+C_{g d}\right)\right]-\omega^{2} R_{g} g_{m} \tau\left(C_{g s}+C_{g d}\right)}{\left[1+\omega^{2} R_{g}^{2}\left(C_{g s}+C_{g d}\right)^{2}\right]\left(1+\omega^{2} \tau^{2}\right)} \\
& \mathrm{Y}_{22}=\mathrm{g}_{\mathrm{ds}}+j \omega\left(C_{g d}+C_{s d}\right)+\frac{\omega^{2} R_{g} C_{g d}{ }^{2}}{1+\omega^{2} R_{g}^{2}\left(C_{g s}+C_{g d}\right)^{2}} \\
& +\frac{j \omega R_{g} g_{m} C_{g d}+\omega^{2} R_{g} g_{m} C_{g d}\left[\tau+R_{g}\left(C_{g s}+C_{g d}\right)\right]}{\left[1+\omega^{2} R_{g}^{2}\left(C_{g s}+C_{g d}\right)^{2}\right]\left(1+\omega^{2} \tau^{2}\right)}
\end{aligned}
$$

Using the real and imaginary parts of $Y$-parameters, the analytical values of the device parameters can be extracted as follows [15]:

$$
\begin{aligned}
g_{m} & =\left.\operatorname{Re}\left[Y_{21}\right]\right|_{\omega^{2}=0} \\
g_{d s} & =\left.\operatorname{Re}\left[Y_{22}\right]\right|_{\omega^{2}=0} \\
R_{g} & =\frac{\operatorname{Re}\left[Y_{11}\right]}{\left(\operatorname{Im}\left[Y_{11}\right]\right)^{2}} \\
C_{g s} & =\frac{\operatorname{Im}\left[Y_{11}\right]+\operatorname{Im}\left[Y_{12}\right]}{\omega} \\
C_{g d} & =-\frac{\operatorname{Im}\left[Y_{12}\right]}{\omega} \\
\tau=- & \frac{\operatorname{Im}\left[Y_{12}\right]}{\omega}+C_{d g}+g_{m} R_{g}\left(C_{g s}+C_{g d}\right) \\
C_{m} & \frac{\operatorname{Im}\left[Y_{22}\right]}{\omega}-C_{g d}-R_{g} g_{m} C_{g d}
\end{aligned}
$$

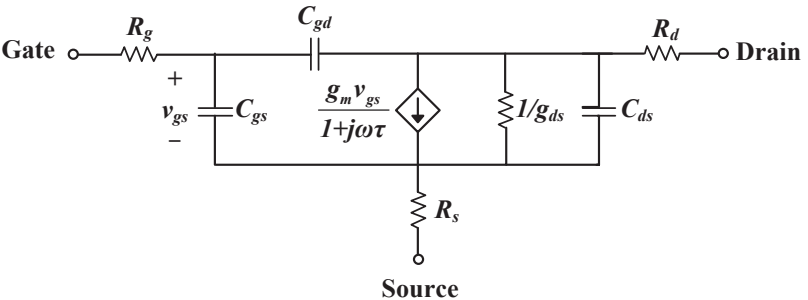

Fig. 2. Nonquasistatic equivalent circuit of a transistor to extract smallsignal parameters of p-n-p-n TFETs by the $Y$-parameter analysis.

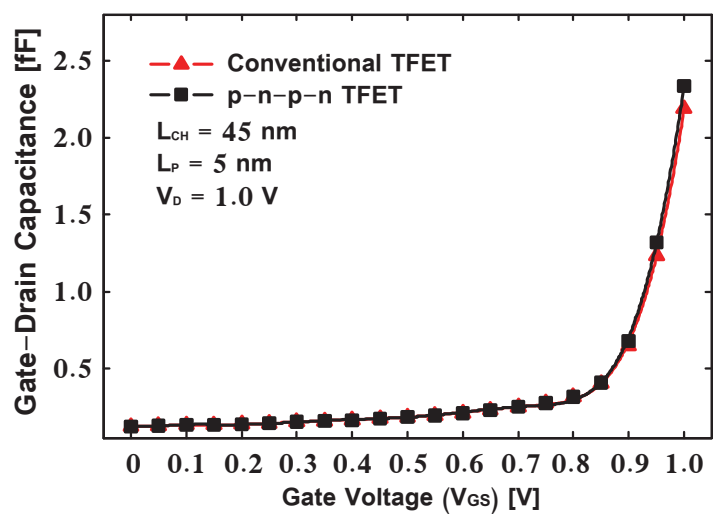

(a)

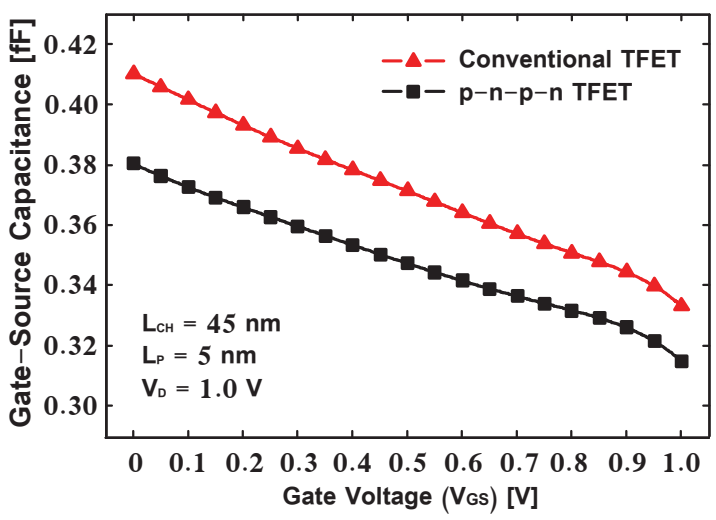

(b)

Fig. 3. Gate capacitance values of the p-n-p-n and conventional TFET as a function of $V_{G S}$. (a) gate-drain capacitance and (b) gate-source capacitance.

Extracted small-signal parameters from TCAD simulations have been used for the evaluation of the RF performances. The cut-off and maximum oscillation frequencies of TFETs have been obtained from the high-frequency current gain and unilateral power gain data of the TCAD simulations, respectively.

\section{Results and Discussion}

The extracted gate-drain and gate-source capacitance of the p-n-p-n and conventional TFETs as a function of the gate voltage $\left(V_{G S}\right)$ are shown in Fig.3. The $C_{g d}$ is responsible for the capacitance between the gate and the inversion layer because of the formation of the inversion layer of a TFET from the drain side toward the source side with increasing $V_{G S}[16]$. Due to 


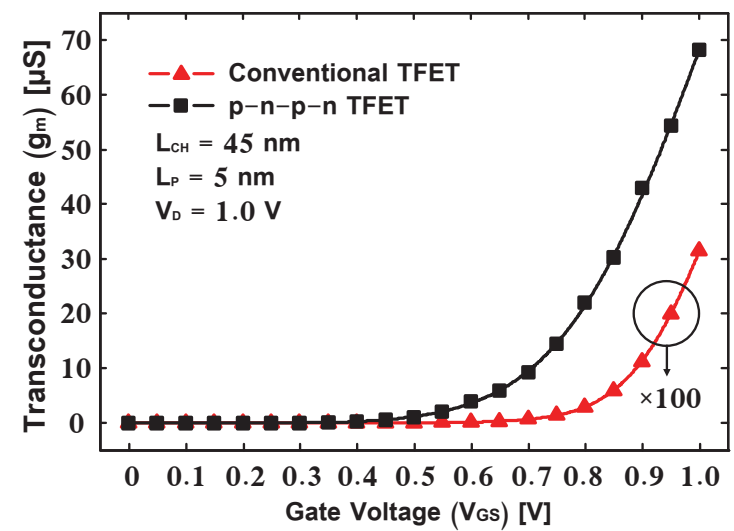

Fig. 4. Comparison of transconductance $\left(g_{m}\right)$ between a p-n-p-n and conventional TFET as a function of gate voltage $\left(V_{G S}\right)$.

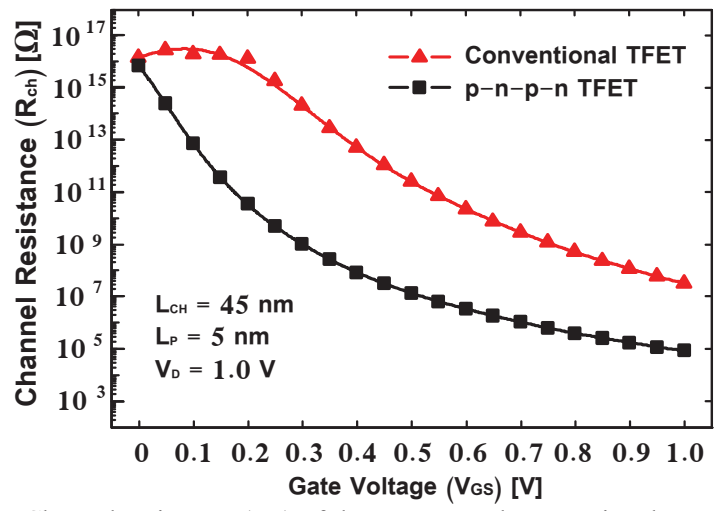

Fig. 5. Channel resistance $\left(R_{c h}\right)$ of the p-n-p-n and conventional TFETs as a function of gate voltage $\left(V_{G S}\right)$.

reduction of the potential barrier at the drain side, increase in $V_{G S}$ causes gate-drain capacitance extremely to increase, as can be confirmed in Fig. 3(a). On the other hand, the gate-source capacitance monotonically decreases with increasing $V_{G S}$, as shown in Fig. 3(b). Extension of the inversion layer from the drain side toward the source side leads to fewer coupling between the gate and the source [16]. In the case of the p-n-p-n TFET, the values of $C_{g s}$ are less than those of the conventional TFET due to the reduction of the barrier width formed at the source-channel junction.

Fig. 4 shows the transconductance $\left(g_{m}\right)$ dependence of p-n$\mathrm{p}-\mathrm{n}$ and conventional TFETs on the gate voltage $\left(V_{G S}\right)$. It has been observed that the $p-n-p-n$ structure has about 200 times higher transconductance than the conventional structure at high $V_{G S}$. Due to a heavily doped $\mathrm{n}^{+}$pocket region at the sourcechannel junction, the barrier width at the source-channel junction reduces. Therefore, improving the lateral electric field appearing at the tunneling junction leads to an increase in the higher ON-current and transconductance [11].

Fig. 5 shows the extracted channel resistance $\left(R_{c h}\right)$ for p-n$\mathrm{p}-\mathrm{n}$ and conventional TFETs as a function of $V_{G S}$. Time constant for the charging delay $\left(R_{c h} C_{g d}\right)$ is an important parameter in the NQS effects which shows how fast the channel charges respond to the input signal. As can be confirmed from Fig. 5, because of the large channel conductivity induced by smaller barrier width and the total

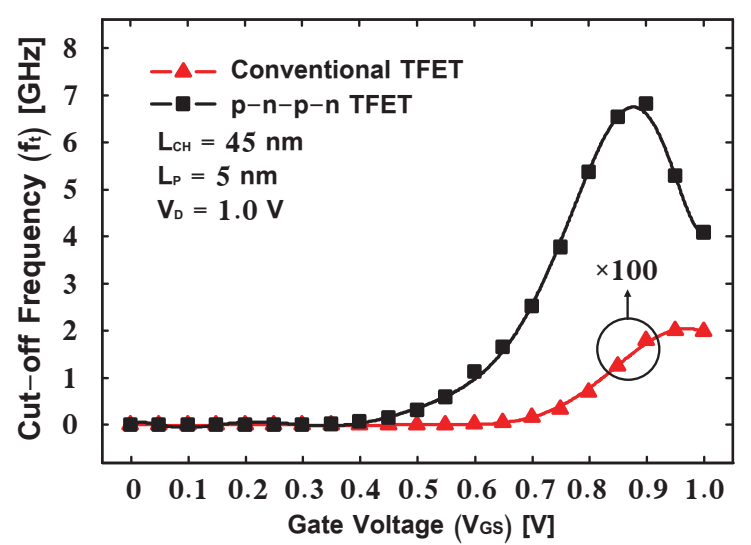

(a)

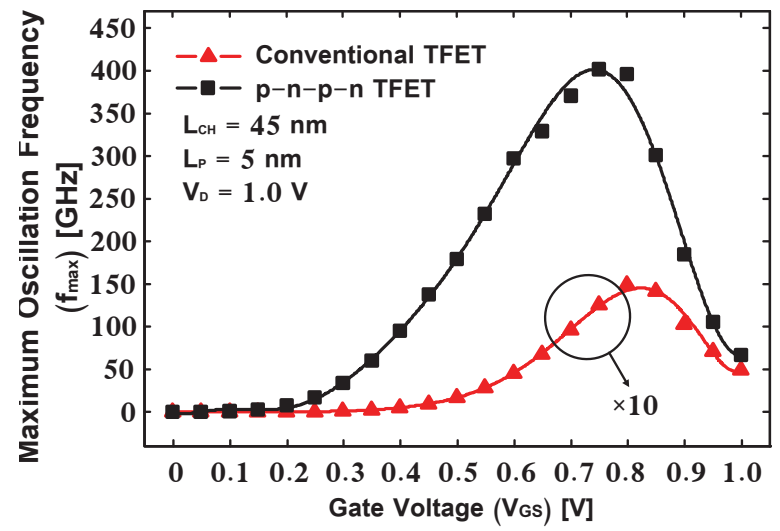

(b)

Fig. 6. Comparison of the (a) $f_{T}$, (b) $f_{\max }$ values between the p-n-p-n TFETs and the conventional TFETs as a function of $V_{G S}$.

band-to-band tunneling rate in the on-state, $R_{c h}$ of $\mathrm{p}-\mathrm{n}-\mathrm{p}-\mathrm{n}$ TFET is much smaller than that of the conventional TFET. Consequently, the switching speed of p-n-p-n TFETs can be higher than that of conventional TFETs.

The RF performance of $p-n-p-n$ TFET is evaluated by extracting the cut-off frequency $\left(f_{T}\right)$ and maximum oscillation frequency $\left(f_{\max }\right)$, known as figures of merit (FoM). The values of the cut-off and maximum oscillation frequencies have been extracted by high-frequency current gain and unilateral power gain using the TCAD-simulated $Y$-parameter data, respectively. Here, $f_{T}$ is extracted when the current gain is unity, and $f_{\max }$ is extracted when Mason's unilateral power gain drops to unity. Fig.6 compares the extracted $f_{T}$ and $f_{\max }$ of the p-n-p-n and conventional TFETs as a function of $V_{G S}$. Generally, the cut-off frequency is related to the $g_{m}, C_{g d}$ and $C_{g s}$. It can be seen from Fig.6 (a) that p-n-p-n structure shows a better $f_{T}$ as well as the switching speed than conventional TFETs because of the higher transconductance and current drivability. However, The $C_{g g}$ (sum of the $C_{g s}$ and $C_{g d}$ ) of a p-n-p-n TFET is nearly similar to conventional TFETs as shown in Figs. 3. The maximum $f_{T}$ values of the p-n-p-n and conventional TFETs are about 6.8 , and $0.020 \mathrm{GHz}$, respectively. The $f_{\max }$ is obtained under power-matching conditions at both the input and output ports drops to unity. As previously shown in Figs. 4, 5 and 6 (a); due to higher $f_{T}$ and $g_{m}$ and lower channel resistance, the p- 
n-p-n TFETs have higher $f_{\max }$ values than conventional TFETs. The maximum $f_{\max }$ values of the extended source and conventional TFETs were about 400, and $15 \mathrm{GHz}$, respectively. The results indicate that $p-n-p-n$ TFETs can have higher cut-off and maximum oscillation frequencies and smaller switching time for high-frequency and high-switchingspeed electronics applications.

\section{VALIDATION OF RADIO-FREQUENCY MODEL}

In order to validate the proposed models, Fig. 7 compares the modeled $Y$-parameters by SPICE simulation with the values obtained from TCAD simulation up to $250 \mathrm{GHz}$, which covers the cut-off and maximum oscillation frequencies. It is observed that $Y$-parameters obtained from the NQS model equivalent circuit show excellent agreement with the calculation results by the TCAD simulation. Consequently, the proposed model in the previous section is valid up to the extremely high frequency range.

\section{CONCLUSION}

An RF model based on parameter extractions for $p-n-p-n$ double-gate TFETs has been presented using TCAD simulations. A p-n-p-n TFET has been compared with the conventional TFETs through analyses of model parameters with variations on bias conditions in terms of the RF performances and switching-speed. Because of the higher transconductance and current drivability and smaller channel resistance of the $p-n-p-n$ TFETs compared to the conventional TFETs, $p-n-p-n$ TFETs can have higher cut-off and maximum oscillation frequencies and smaller switching time. The RF model was well matched with the $Y$-parameters induced from the TCAD simulation up to the extremely high frequency range for the millimeter-wave applications.

\section{REFERENCES}

[1] A. M. Ionescu and H. Riel, "Tunnel field-effect transistors as energyefficient electronic switches," Nature, vol. 479, no. 7373, pp. 329-337, 2011.

[2] E. Baravelli, E. Gnani, R. Grassi, A. Gnudi, S. Reggiani and G. Baccarani, "Optimization of $\mathrm{n}$ - and $\mathrm{p}$-type TFETs integrated on the same InAs/ $\mathrm{Al}_{\mathrm{x}} \mathrm{Ga}_{1-\mathrm{x}} \mathrm{Sb}$ technology platform," IEEE Trans. Electron Devices, vol. 61, no. 1, pp. 178-185, 2014.

[3] W. Wang, P.-F. Wang, C.-M. Zhang, X. Lin, X.-Y. Liu, Q.-Q. Sun, P. Zhou and D. W. Zhang, "Design of U-shape channel tunnel FETs with SiGe source regions," IEEE Trans. Electron Devices, vol. 61, no. 1, pp. 193-197, 2014.

[4] K. Ganapathi and S. Salahuddin, "Heterojunction vertical band-to-band tunneling transistors for steep subthreshold swing and high ON current," IEEE Electron Device Lett., vol. 32, no. 5, pp. 689-691, 2011.

[5] L. Britnell, R. V. Gorbachev, R. Jalil, B. D. Belle, F. Schedin, A. Mishchenko, T. Georgiou, M. I. Katsnelson, L. Eaves, S. V. Morozov, N. M. R. Peres, J. Leist, A. K. Geim, K. S. Novoselov, L. A. Ponomarenko, "Field-effect tunneling transistor based on vertical graphene heterostructures," Science, vol. 335, no. 6071, pp. 947-950, 2012.

[6] K-T. Lam, X. Cao, and J. Guo, "Device Performance of Heterojunction Tunneling Field-Effect Transistors Based on Transition Metal Dichalcogenide Monolayer," IEEE Electron Device Lett., vol. 34, no. 10, pp. 1331-1333, 2013.

[7] K. Boucart and A. M. Ionescu, "Double-gate tunnel FET with high-k gate dielectric," IEEE Trans. Electron Devices, vol. 54, no. 7, pp. 1725$1733,2007$.

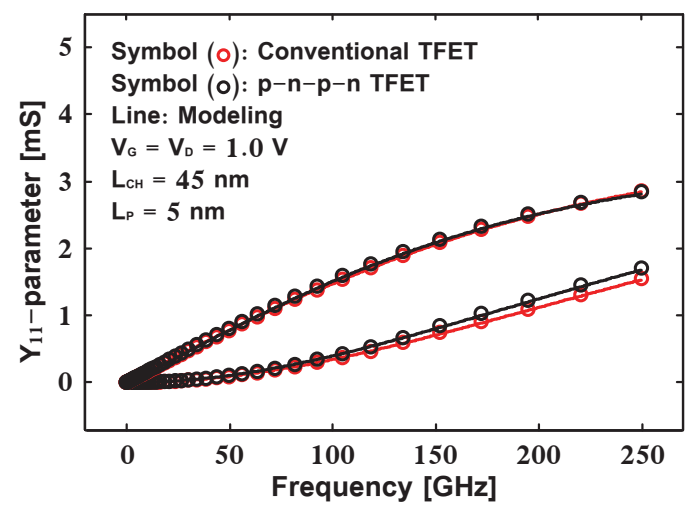

(a)

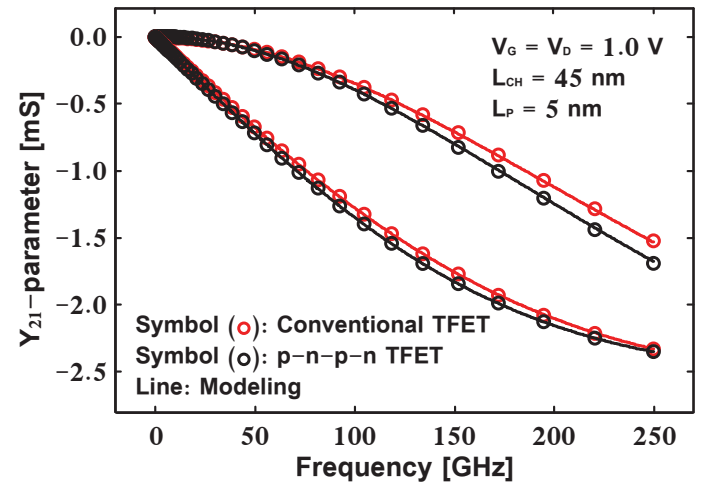

(b)

Fig. 7. Comparison of modeled (line) and values obtained from TCAD simulation (symbol) $Y$-parameters of p-n-p-n and conventional TFET at $V_{G S}$ $=V_{D S}=1.0 \mathrm{~V}$. (a) $Y_{11}$ and (b) $Y_{21}$.

[8] H. Chang, B. Adams, P. Chien, J. Li, and J. C. S. Woo, "Improved Subthreshold and Output Characteristics of Source-Pocket Si Tunnel FET by the Application of Laser Annealing," IEEE Trans. Electron Devices, vol. 60, no. 1, pp. 92-96, 2013.

[9] S. Marjani and S. E. Hosseini, "A Novel Double Gate Tunnel Field Effect Transistor with $9 \mathrm{mV} / \mathrm{dec}$ Average Subthreshold Slope," presented at the $22^{\text {st }}$ Iranian Conference on Electrical Engineering (ICEE 2014), Tehran, Iran, 2014.

[10] S. Marjani and S. E. Hosseini, "Radio-Frequency Modeling of SquareShaped Extended Source Tunneling Field-Effect Transistors," Superlattices and Microstructures, vol. 76, pp. 297-314, 2014.

[11] V. Nagavarapu, R. Jhaveri, and J. C. S. Woo, "The Tunnel Source (PNPN) n MOSFET: A Novel High Performance Transistor," IEEE Trans. Electron Devices, vol. 55, no. 4, pp. 1013-1019, 2008.

[12] R. Narang, M. Saxena, R. S. Gupta and M. Gupta, "Drain current model for a gate all around (GAA) p-n-p-n tunnel FET," Microelectronics Journal, vol. 44, no. 6, pp. 479-488, 2013.

[13] ATLAS Device Simulation Software, Silvaco Int., Santa Clara, CA, USA, 2012.

[14] B. Ghosh and M. W. Akram, "Junctionless tunnel field effect transistor", IEEE Electron Dev. Lett., vol. 34, no. 5, pp. 584-586, 2013.

[15] I. Kwon, M. Je, K. Lee and H. Shin, "A simple and analytical parameterextraction method of a microwave MOSFET," IEEE Trans. Microwave Theory Tech., vol. 50, no. 6, pp. 1503-1509, 2002.

[16] Y. Yang, X. Tong, L.-T. Yang, P.-F. Guo, L. Fan, and Y.-C. Yeo "Tunneling field-effect transistor: capacitance components and modeling," IEEE Electron Dev. Lett., Vol. 31, No. 7, pp. 752-754, 2010. 